Check for updates

Cite this: RSC Adv., 2018, 8, 34514

Received 13th July 2018

Accepted 18th September 2018

DOI: 10.1039/c8ra05992f

rsc.li/rsc-advances

\section{A fast screening model for drug permeability assessment based on native small intestinal extracellular matrix}

\author{
Na Li,$\uparrow^{\mathrm{ab}}$ Zhigang Sui, $\dagger^{\mathrm{c}}$ Yong Liu, ${ }^{d}$ Dandan Wang, ${ }^{a}$ Guangbo Ge (D) *a \\ and Ling Yang $(\mathbb{D}$ *a
}

The Caco-2 cell monolayer model is widely utilized to predict drug permeability across human intestinal epithelial cells. However, at least 21 days is required for the formation and maturation of a well-tight Caco-2 cell monolayer, thereby restricting the throughput of the screening model during drug discovery. To address this challenge, a fast (7 days), and more physiologically relevant screening model integrating both the Caco-2 cell model and a small intestinal submucosa (SIS) hydrogel was developed in this study. The 7 day model exhibited desirable phenotype and functional similarity to the conventional 21 day Caco-2 model with respect to paracellular resistance, alkaline phosphatase (ALP) activities, and the mRNA expression level of three transporters (PEPT1, OATP1A2, and P-gp) as well as their mediated influx or efflux. Besides, the increased gene expression of two excretive transporters (BCRP, MRP2) and their enhanced functionality were observed in the current fast model compared to the traditional 21 day model. More importantly, a strong correlation $\left(r^{2}=0.9458\right)$ was obtained between the absorptive $P_{\text {app }}$ values of 19 model compounds in the 7 day model and those in the conventional 21 day model. These results revealed the pivotal role of the native extracellular matrix (SIS) in facilitating the differentiation of Caco-2 cells, leading to the reconstruction of the accelerated 7 day model, which presents a promising tool for screening drug permeability in future drug discovery.

\section{Introduction}

Oral administration is the most popular route for drug delivery due to its relative convenience, safety, and patient compliance..$^{1,2}$ The small intestine is the major absorption site of oral drugs, ${ }^{3,4}$ so poor intestinal absorption is prone to cause higher probabilities of failure in the early phases of drug discovery; ${ }^{5}$ hence, the accurate evaluation of oral drug absorption in vitro is pivotal for drug development. ${ }^{6}$ In spite of the colorectal adenocarcinoma origin, the Caco-2 cell monolayer exhibits lots of features associated with absorptive intestinal epithelial cells, such as polarized differentiation, high levels of expression of brush border enzymes and transporters, and well-formed tight junctions, etc $^{7-9}$ Therefore, it is believed to be reliable to predict the intestinal absorption of new chemical entities in humans

${ }^{a}$ Institute of Interdisciplinary Integrative Medicine Research, Shanghai University of Traditional Chinese Medicine, Shanghai 201203, China. E-mail: geguangbo@ shutcm.edu.cn; yling@dicp.ac.cn

${ }^{b}$ Department of Biomedical Engineering, University of South Dakota, SD, 57107, USA 'Dalian Institute of Chemical Physics, Chinese Academy of Sciences, Dalian, 116023, China

${ }^{d}$ School of Life Science and Medicine, Dalian University of Technology, Panjin, 124221, China

$\dagger$ These authors contributed equally to this work. from their membrane permeability across the Caco-2 cell monolayer. ${ }^{10,11}$ However, it is difficult to accomplish highthroughput screening with the traditional Caco-2 cell model due to its long culturing period (21 days) allowing for full cell differentiation into the enterocyte-like phenotype. ${ }^{12}$ Moreover, the 21 day culture necessary for Caco-2 cells not only increases the probability of contamination but also brings undesirable high costs for drug discovery. ${ }^{13}$

To provide a more cost-effective high-throughput screening model for the pharmaceutical industry, several strategies have been proposed to accelerate the differentiation process of Caco2 cells, including coating the transwell insert with collagen, increasing the cell seeding density, and utilizing differentiationinducing agents such as butyric acid, growth factors and hormones, etc. ${ }^{14,15}$ Although to some extent these protocols are able to speed up the Caco-2 monolayer maturation process, the additive factors make the Caco-2 cell model more sophisticated and cost-consuming. More importantly, Caco-2 cells grown in these conditions are reported to express fewer tight junction proteins and efflux pumps, which restrict their usefulness in predicting paracellular permeability and the active transport of drug candidates. ${ }^{13}$ Thus, it is imperative to develop a rapid screening model based on the Caco-2 cell model while keeping all the transport functions of Caco-2 cells. 
Distinct from the above-mentioned procedures to accelerate phenotype and functionality development of Caco-2 cells, physiological microenvironment conditioning is another effective pathway to facilitate cell differentiation. ${ }^{16}$ Extracellular matrix (ECM) and stromal cells are the major microenvironment constituents surrounding intestinal epithelial cells, thereby eliciting universal cell-ECM and cell-cell interactions that exist in all kinds of tissues and organs. ${ }^{17}$ It has been widely demonstrated that such interactions play a crucial role during the organization and maintenance of all tissues and organs. ${ }^{18,19}$ Correspondingly, Visco et al. reported that human fibroblasts could induce the proliferation and differentiation of intestinal epithelial cells through the direct paracrine action of keratinocyte growth factor. ${ }^{20}$ Small intestinal submucosa (SIS), the native ECM in the small intestine, is composed of a variety of biological signaling molecules, including collagen, glycoproteins, glycosaminoglycans, and growth factors, etc. ${ }^{21-23}$ Accumulating evidence indicates that decellularized SIS could provide beneficial biochemical and biophysical cues for various types of cells, thereby promoting cell attachment, proliferation, migration, and differentiation. ${ }^{24}$ Furthermore, SIS has been broadly applied in the construction of different tissue grafts and subsequent reconstructive therapies. ${ }^{25}$ Nevertheless, the exact effect of this biological ECM derived from the small intestine on Caco-2 cell differentiation is still unknown. Therefore, it is reasonable to believe that the combination of SIS and the Caco2 cell model might provide the possibility for the implementation of a novel fast screening model for drug permeability evaluation.

In the current study, we tested the hypothesis that the microenvironment provided by biological SIS would effectively facilitate Caco-2 cell differentiation towards the mature phenotype of intestinal epithelial cells through cell-ECM communication, consequently accelerating the formation of a functional Caco-2 cell monolayer. In this context, the SIS hydrogel was incorporated into the Caco-2 cell model to construct a modified 7 day screening model, which was compared with the traditional 21 day Caco- 2 cell model in terms of proliferation ability, alkaline phosphatase activity, tightness of the junctions, and active transport as well as metabolism enzyme activity. Finally, the absorptive permeability of 19 marketed drugs covering a wide range of absorption characteristics and clinical applications were determined with both the 21 day model and the 7 day fast model, so that the correlation analysis between them could be performed. We hope this optimized model, possessing both high throughput and potential functionality, could work as an ideal alternative to the conventional Caco-2 cell model for accurate drug absorption prediction.

\section{Materials and methods}

\section{Decellularization of porcine small intestine and H\&E staining}

All animal procedures in this study were performed in compliance with the Guidelines for Care and Use of Laboratory Animals of Dalian Medical University, and the experiments were approved by the Animal Ethics Committee of Dalian Medical
University. Porcine small intestine was obtained from healthy market pigs $(\sim 200 \mathrm{~kg})$ within $4 \mathrm{~h}$ of sacrifice and cut into segments with $20 \mathrm{~cm}$ length. After cleaning with phosphate buffered saline (PBS), jejunum segments were subjected to decellularization according to the protocol described previously. ${ }^{26,27}$ In brief, tunica serosa, tunica muscularis and tunica mucosa were mechanically removed from the small intestine successively, followed by thoroughly rinsing with PBS. After that, the left tunica submucosa with basilar portions of the tunica mucosa was treated with $0.1 \%$ peracetic acid (Sigma, St. Louis, MO) for $30 \mathrm{~min}$ to remove the remaining cells, DNA and RNA, and then washed thoroughly with PBS. The decellularized SIS scaffolds were stored at $-80{ }^{\circ} \mathrm{C}$.

Fresh jejunum segments and decellularized SIS scaffolds were fixed with $4 \%$ paraformaldehyde (Affymetrix, CA) at $4{ }^{\circ} \mathrm{C}$ overnight, followed by washing three times with PBS. The paraffin-embedded sections ( $5 \mu \mathrm{m}$ thickness) were prepared by the 2nd Medical Center of Dalian Medical University (Dalian, China) and stained with hematoxylin and eosin dyes (H\&E; EMD Chemicals). Images were acquired using an Olympus microscope (CKX41).

\section{Preparation of SIS hydrogel and construction of the fast model}

The acellular SIS scaffolds were ground in liquid nitrogen and subjected to enzyme digestion with $0.1 \%$ pepsin dissolved in $3 \%$ acetic acid under mechanical agitation for $48 \mathrm{~h}$. Then, the SIS suspension was centrifuged at $13000 \mathrm{rpm}$ for $30 \mathrm{~min}$. Subsequently, the supernatant was lyophilized and sterilized under UV for $1 \mathrm{~h}$. Finally, the swelled SIS was dissolved in $0.2 \%$ acetic acid for construction of the fast absorption model.

Human colon adenocarcinoma cells Caco-2 (passage 45-55; Cat. no. HTB-37) were purchased from ATCC. Neutral SIS gel solution $\left(2 \mathrm{mg} \mathrm{mL}{ }^{-1}\right)$ was pipetted onto the top of 12-well transwell filter inserts (Cat. no. 3460, Corning) and allowed to polymerize for $2 \mathrm{~h}$ at $37^{\circ} \mathrm{C}$. Thereafter, $1.5 \times 10^{5}$ Caco-2 cells were seeded onto the surface of solidified SIS gel and cultured for 7 days (Fig. 2a). Caco-2 cells at the same density were directly added to each transwell insert and cultured for 21 days, serving as the control (the traditional Caco-2 cell model). All the cells were maintained in the high-glucose Dulbecco's modified Eagle's medium containing 10\% fetal bovine serum (FBS), 1\% nonessential amino acid, and 1\% penicillin/streptomycin (all from Invitrogen) at $37{ }^{\circ} \mathrm{C}$ in a $5 \% \mathrm{CO}_{2}$ humidified incubator, and the medium was replaced every other day.

\section{Cell viability and scanning electron microscopy (SEM) examination}

To assess cell viability in the fast model, Caco-2 cells grown on the SIS hydrogel for 7 days were stained with calcein-AM/EthD-1 solution (Invitrogen) as described previously. ${ }^{5}$ The samples were observed using confocal microscopy (Leica SP2). Live cells were stained green because of ubiquitous intracellular esterase activity that can digest nonfluorescent calcein-AM into green fluorescent calcein, whereas dead cells were stained red due to the EthD-1 entrance through damaged membranes and the emission of red fluorescence upon binding to nucleic acids. 
The decellularized SIS scaffolds were fixed with $2.5 \%$ glutaraldehyde (Sigma) overnight at $4{ }^{\circ} \mathrm{C}$ and dehydrated in a series of graded ethanol $(25 \%, 50 \%, 75 \%, 95 \%$ and $100 \%)$ before lyophilization. The dried samples were then mounted onto stubs, sputtered with platinum, and examined with SEM (XL-30; Philips-FEI).

\section{Proliferation ability and alkaline phosphatase activity measurement}

To compare the proliferation ability of Caco-2 cells in the fast model and the traditional 21 day model, samples were harvested and subjected to DNA quantification analysis at indicated time points as described previously. ${ }^{5}$ Briefly, the DNA contents were measured using PicoGreen DNA assay following the protocol provided by the manufacturer (Molecular Probe), and analyzed by a microplate reader (Synergy ${ }^{\mathrm{TM}} \mathrm{H} 1$, BioTek ${ }^{\circledR}$ Instruments, USA) at an excitation wavelength of $480 \mathrm{~nm}$ and an emission wavelength of $530 \mathrm{~nm}$.

The alkaline phosphatase (ALP) activities of Caco-2 cells in both the fast model and 21 day model were determined according to the protocol reported previously with minor modifications ${ }^{28}{ }^{28}$ in brief, the cells grown on SIS gel or transwell insert were washed twice with PBS and then incubated with $1 \mathrm{mg} \mathrm{mL}{ }^{-1}$ of $p$-nitrophenyl phosphate ( $p$-NPP) in $0.2 \mathrm{M}$ Tris buffer (Sigma) at $37^{\circ} \mathrm{C}$. At different time points $(5 \mathrm{~min}, 10 \mathrm{~min}$, $15 \mathrm{~min}, 20 \mathrm{~min}$ ), $200 \mu \mathrm{L}$ of $3 \mathrm{M} \mathrm{NaOH}$ were added to each well to stop the reaction. The released $p$-nitrophenol ( $p$-NP) content in the supernatant was determined at $405 \mathrm{~nm}$ with a microplate reader. The cellular protein amount in each sample was quantitatively analyzed using the BCA assay kit (Beyotime, Beijing, China) to standardize the $p$-NP concentration measured above.
The ALP activities of all samples were normalized to that of the 21 day control model after reaction with $p$-NPP for $5 \mathrm{~min}$.

\section{Real-time RT-PCR analysis}

To assess the transcript expression level of intercellular junction proteins (ZO-1, occludin, E-cadherin, claudin-1), transporters (OATP1A2, PEPT1, P-gp, MRP2, BCRP), and metabolism enzyme (UGT1A1) in both the fast model and the 21 day control model, total RNA was extracted using RNAiso Plus reagent (Takara, Dalian) according to the manufacturer's instruction, and quantified by spectrophotometric analysis at $260 \mathrm{~nm}$. Subsequently, a portion of $500 \mathrm{ng}$ RNA underwent reverse transcription in a $10 \mu \mathrm{L}$ reaction mixture using RT-PCR kit (Takara, Dalian). Lastly, real-time PCR was conducted with a SYBR ${ }^{\circledR}$ Premix Ex Taq $^{\text {TM }}$ II Kit (Takara, Dalian) using the StepOne ${ }^{\mathrm{TM}}$ Real-Time PCR System (Applied Biosystems, Foster City, CA). The primer sequences for real-time PCR are listed in Table 1. The relative expression level for each target gene was normalized by the CT value of GAPDH ( $2 \Delta \mathrm{CT}$ formula). Each sample was performed in triplicate.

\section{Functional characterization}

The integrity of Caco-2 cell monolayers formed in both the fast model and 21 day control model was monitored by TEER measurement with a Millicell-ERS-electrode (Millipore, Billerica, MA). Only cultures with TEER values higher than $400 \Omega$ $\mathrm{cm}^{2}$ were employed in further transport experiments.

The absorptive permeability of marker compounds (all from Sigma) for paracellular passive transport (fluorescein), active transport (fexofenadine, cephalexin, digoxin, sulfasalazine, estradiol-17-G), and transcellular passive transport (listed in Table 2) were measured as described in our previous study. ${ }^{38}$

Table 1 List of primers used in the real-time RT-PCR assay

\begin{tabular}{|c|c|c|}
\hline Gene & Primer sequence & Reference \\
\hline \multirow[t]{2}{*}{ ZO-1 } & Forward 5'-ATGGTGTCCTACCTAATTCAACTCAT-3' & 29 \\
\hline & Reverse 5'-GCCAGCTACAAATATTCCAACATCA-3' & \\
\hline Occludin & Reverse $5^{\prime}$-TATTCCCTGATCCAGTCCTCCTC-3' & \\
\hline \multirow[t]{2}{*}{ E-cadherin } & Forward 5'-CAGGCTCAAGCTATCCTTGC- $3^{\prime}$ & 30 \\
\hline & Reverse 5'-AGTCATGCGTAGTGGTGCAT-3' & \\
\hline \multirow[t]{2}{*}{ OATP1A2 } & Forward 5'-AAGACCAACGCAGGATCCAT-3' & 32 \\
\hline & Reverse 5'-GAGTTTCACCCATTCCACGTACA-3' & \\
\hline \multirow[t]{2}{*}{ PEPT1 } & Forward 5'-AATGTTCTGGGCCTTGTTTG-3' & 33 \\
\hline & Reverse 5'-CATCTGATCGGGCTGAATTT-3' & \\
\hline \multirow[t]{2}{*}{ P-gp } & Forward 5'-CCCATCATTGCATATGCAGG-3' & 34 \\
\hline & Reverse $5^{\prime}$-GTTCAAACTTCTACTCCTGA-3' & \\
\hline \multirow[t]{2}{*}{ UGT1A1 } & Forward 5'-GGTGACTGTCCAGGACCTATTGA-3' & 36 \\
\hline & Reverse $5^{\prime}$-TAGTGGATTTTGGTGAAGGCAGTT-3' & \\
\hline \multirow[t]{2}{*}{ GAPDH } & Forward 5'-CTCTCTGCTCCTCCTGTTCG-3' & 37 \\
\hline & Reverse $5^{\prime}$-GCCCAATACGACCAAATCC- $3^{\prime}$ & \\
\hline
\end{tabular}


Table 2 Validation set of model compounds

\begin{tabular}{|c|c|c|c|c|c|c|}
\hline No. & Compound & $\begin{array}{l}\text { Primary transport } \\
\text { mechanism }\end{array}$ & $\mathrm{FA}^{a}(\%)$ & $\begin{array}{l}P_{\text {app }}(\mathrm{A}-\mathrm{B}) \times 10^{-6}\left(\mathrm{~cm} \mathrm{~s}^{-1}\right) \\
21 \text { day control }\end{array}$ & $\begin{array}{l}P_{\text {app }}(\mathrm{A}-\mathrm{B}) \times 10^{-6}\left(\mathrm{~cm} \mathrm{~s}^{-1}\right) \\
\text { fast model }\end{array}$ & Clinical application \\
\hline 1 & Reserpine & PT & 50 & $3.50 \pm 0.69$ & $3.60 \pm 0.43$ & Antihypertensive \\
\hline 2 & Atenolol & PP & 50 & $3.41 \pm 0.23$ & $4.36 \pm 0.68$ & Antihypertensive \\
\hline 3 & Sulfadiazine & PT & 65 & $9.76 \pm 1.05$ & $9.78 \pm 1.44$ & Anti-infective \\
\hline 4 & Terbutaline & PP & 73 & $15.61 \pm 1.28$ & $16.55 \pm 0.70$ & Adrenergic $\beta$-agonist \\
\hline 5 & Ketoconazole & PT & 76 & $9.61 \pm 0.01$ & $8.33 \pm 0.93$ & Antifungal \\
\hline 6 & Omeprazole & $\mathrm{PT}$ & 80 & $12.58 \pm 2.01$ & $11.43 \pm 0.47$ & Anti-ulcer \\
\hline 7 & Ethionamide & PT and/or PP & 80 & $23.75 \pm 0.94$ & $20.45 \pm 5.50$ & Antitubercular \\
\hline 8 & Bupropion & $\mathrm{PT}$ and/or PP & 87 & $16.64 \pm 0.99$ & $12.89 \pm 0.48$ & Antidepressive \\
\hline 9 & Felodipine & PT & 88 & $4.34 \pm 0.63$ & $4.34 \pm 0.10$ & Antihypertensive \\
\hline 10 & Propranolol & PT & 90 & $10.58 \pm 1.66$ & $9.37 \pm 0.03$ & Antihypertensive \\
\hline 11 & Chloramphenicol & PT and/or PP & 90 & $8.17 \pm 0.06$ & $7.12 \pm 0.04$ & Anti-bacterial \\
\hline 12 & Metoprolol & $\mathrm{PT}$ & 95 & $12.05 \pm 0.39$ & $11.82 \pm 0.25$ & Antihypertensive \\
\hline 13 & Verapamil & PT & 95 & $10.05 \pm 0.10$ & $9.70 \pm 1.70$ & Anti-arrhythmia \\
\hline 14 & Lamotrigine & PT and/or PP & 98 & $18.49 \pm 2.84$ & $17.70 \pm 1.14$ & Antiepileptic \\
\hline 15 & Warfarin sodium & PT & 98 & $15.31 \pm 0.002$ & $14.44 \pm 0.11$ & Anticoagulant \\
\hline 16 & Naproxen & PT & 99 & $15.01 \pm 2.37$ & $14.73 \pm 0.81$ & Anti-inflammatory \\
\hline 17 & Nifedipine & PT & 100 & $10.69 \pm 0.11$ & $11.69 \pm 0.53$ & Antihypertensive \\
\hline 18 & Ketoprofen & PT & 100 & $19.19 \pm 1.93$ & $15.04 \pm 1.97$ & Anti-inflammatory \\
\hline 19 & Carbamazepine & PT & 100 & $20.85 \pm 1.93$ & $19.54 \pm 0.87$ & Antimaniacal \\
\hline
\end{tabular}

${ }^{a}$ Human absorption fraction (FA) was obtained from the literature. ${ }^{40}$

Briefly, cell monolayers in the fast model or the conventional control model were washed twice, followed by preincubation with Hank's balanced salt solution (HBSS) at $37{ }^{\circ} \mathrm{C}$ for $15 \mathrm{~min}$. The transport experiments were initiated by replacing the transport buffer in the upper compartment with $25 \mu \mathrm{M}$ model compounds dissolved in HBSS. For each sample, $100 \mu \mathrm{L}$ were taken from the lower compartment at different time points followed by prewarmed HBSS $(100 \mu \mathrm{L})$ supplement. All the samples were diluted with an equal volume of methanol and centrifuged at $14000 \mathrm{rpm}$ for $15 \mathrm{~min}$ before loading into an ultra-fast liquid chromatography system (UFLC, Shimadzu, Kyoto, Japan) with diode array detection. The amounts of fluorescein in the samples were measured using a microplate reader at an excitation wavelength of $488 \mathrm{~nm}$ and an emission wavelength of $530 \mathrm{~nm}$. Apparent permeability coefficients $\left(P_{\text {app }}\right)$ of compounds were calculated according to the following equation:

$$
P_{\text {app }}=\Delta Q /\left(\Delta t A C_{0}\right),
$$

where $P_{\text {app }}$ is the apparent permeability coefficient $\left(\mathrm{cm} \mathrm{s}^{-1}\right), \Delta Q /$ $\Delta t$ indicates the permeability rate $\left(\mu \mathrm{mol} \mathrm{s}^{-1}\right), A$ represents the diffusion area of the monolayers $\left(\mathrm{cm}^{2}\right)$, and $C_{0}$ is the initial concentration of the substance in the donor compartment $(\mu \mathrm{M})$.

The glucuronosyltransferase (UGT) activity assay was performed as reported by Bothe et al. ${ }^{39}$ The cells cultured in the fast model or 21 day model were washed twice with PBS and incubated with $100 \mu \mathrm{M}$ 4-methylumbelliferone (4-MU) in serum-free medium. After $10 \mathrm{~min}, 10 \mu \mathrm{L}$ of supernatant was collected and mixed with $190 \mu \mathrm{L}$ of $10 \mathrm{mM} \mathrm{NaOH}$. The fluorescence intensity of all samples was measured by a microplate reader at an excitation wavelength of $390 \mathrm{~nm}$ and an emission wavelength of $460 \mathrm{~nm}$. The UGT activities were expressed as decreased amounts of 4-MU (pmol) per mg protein.

\section{Statistical analysis}

All reported values were averaged $(n=3)$ and presented as mean \pm standard deviation (SD). Statistical differences were analyzed with Student's two-tailed $t$-test, assuming equal variances, and the value of $P<0.05$ was considered statistically significant.

\section{Results}

\section{Morphological characteristics of decellularized SIS}

As shown in Fig. 1, a thin layer of intact SIS (Fig. 1b and c) was preserved after the native porcine small intestine (Fig. 1a) was subjected to a series of decellularization procedures. HE staining of both the native small intestine and decellularized SIS was performed to examine the decellularization efficiency and the results showed that both the epithelial layer and muscle layer were displayed together with SIS in the native small intestine (Fig. 1d), whereas only SIS was visible after decellularization treatment (Fig. 1e). Macroscopically, the mucosal surface of the longitudinally cut SIS sheet appeared to be more compact than the serosal surface (Fig. 1f versus Fig. 1g). Correspondingly, SEM analysis also revealed the disparity in the topography of the mucosal and serosal surfaces of acellular SIS. Compared with the relatively smooth mucosal surface (Fig. 1h), the more ragged appearance of the serosal side (Fig. 1j) was observed from lowmagnification of SEM images. Furthermore, more detailed ultrastructures of decellularized SIS were demonstrated in highmagnification SEM images. The mucosal surface was characterized by more densely packed collagen fibers (Fig. 1i), whereas 

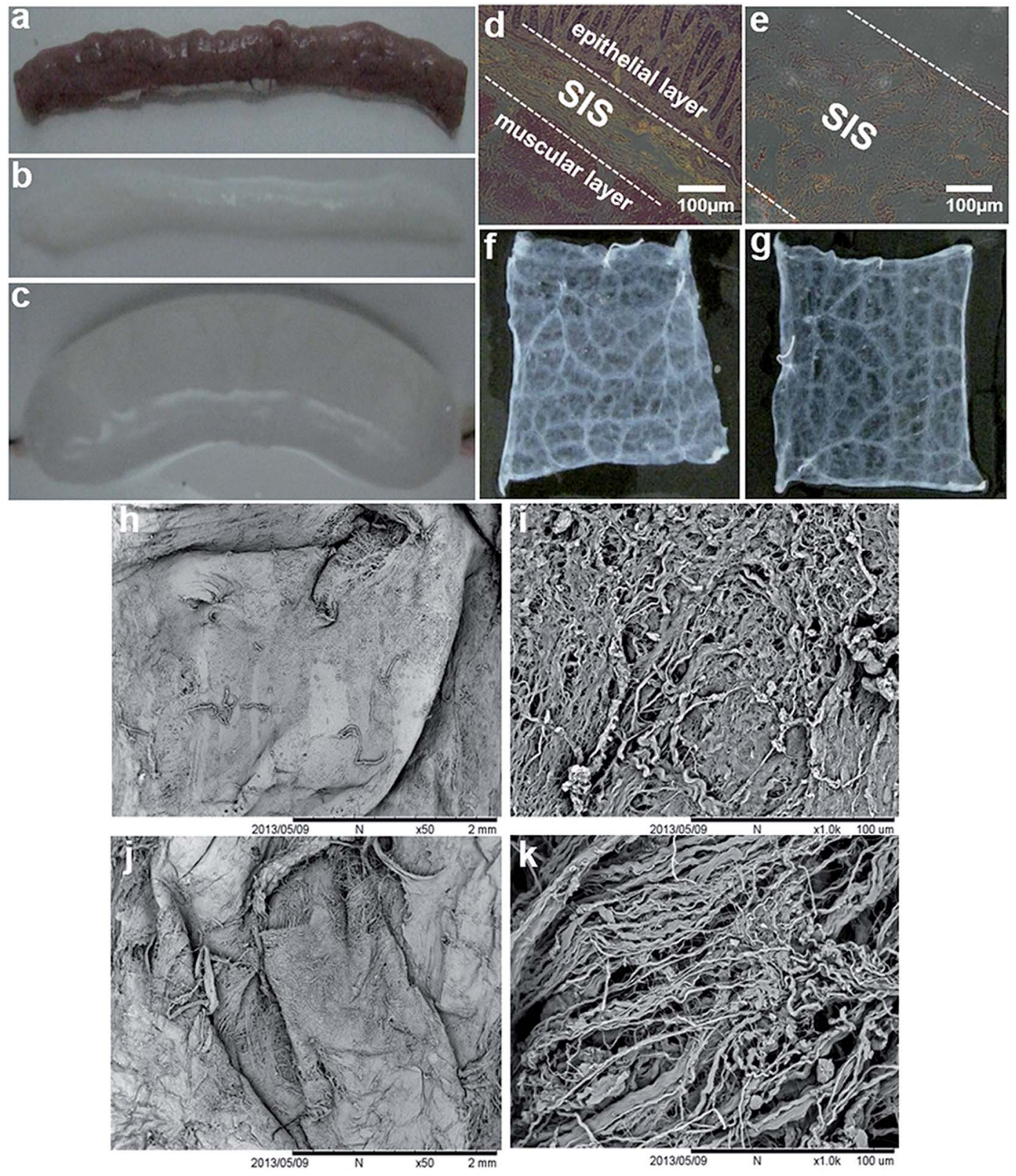

Fig. 1 Characterization of the decellularized SIS scaffolds. A thin layer of intact SIS ( $b$ and c) was preserved after the native porcine small intestine (a) was subjected to decellularization. HE staining of the native small intestine (d) showed SIS was in between the epithelial layer and the muscle layer, whereas only SIS could be visible after decellularization treatment (e). Macroscopically, the mucosal surface (f) of the longitudinally cut SIS sheet appeared to be more compact than the serosal surface (g). Compared with the relatively smooth mucosal surface (h) of SIS, the more ragged appearance of the serosal side (j) was displayed by low-magnification of SEM images. High-magnification of SEM images exhibited the more densely packed collagen fibers on the mucosal surface of SIS (i), whereas a network of loosely organized fibers was present in the serosal surface $(\mathrm{k})$

a network of loosely organized fibers was present in the serosal surface (Fig. 1k).

\section{Morphology and cell viability of the newly developed model}

After three days of cultivation, Caco-2 cells seeded on the surface of SIS hydrogel were observed to be completely confluent under phase contrast microscopy (Fig. 2b). Moreover, a dense cell monolayer was generated on the top of the SIS hydrogel incorporated in the fast model on the seventh day
(Fig. 2c), indicating that the natural SIS material is favorable for Caco-2 cell attachment and growth. To further evaluate the cellular viability of the fast model, Caco- 2 cells grown on the SIS hydrogel were subjected to live/dead staining and the results showed that Caco-2 cells displayed good viability after one week of cultivation (Fig. 2d). This suggests that the SIS hydrogel integrated into the fast absorption model could provide a beneficial microenvironment to maintain Caco- 2 cell viability. In addition, the uniform topology of the epithelial layer was 

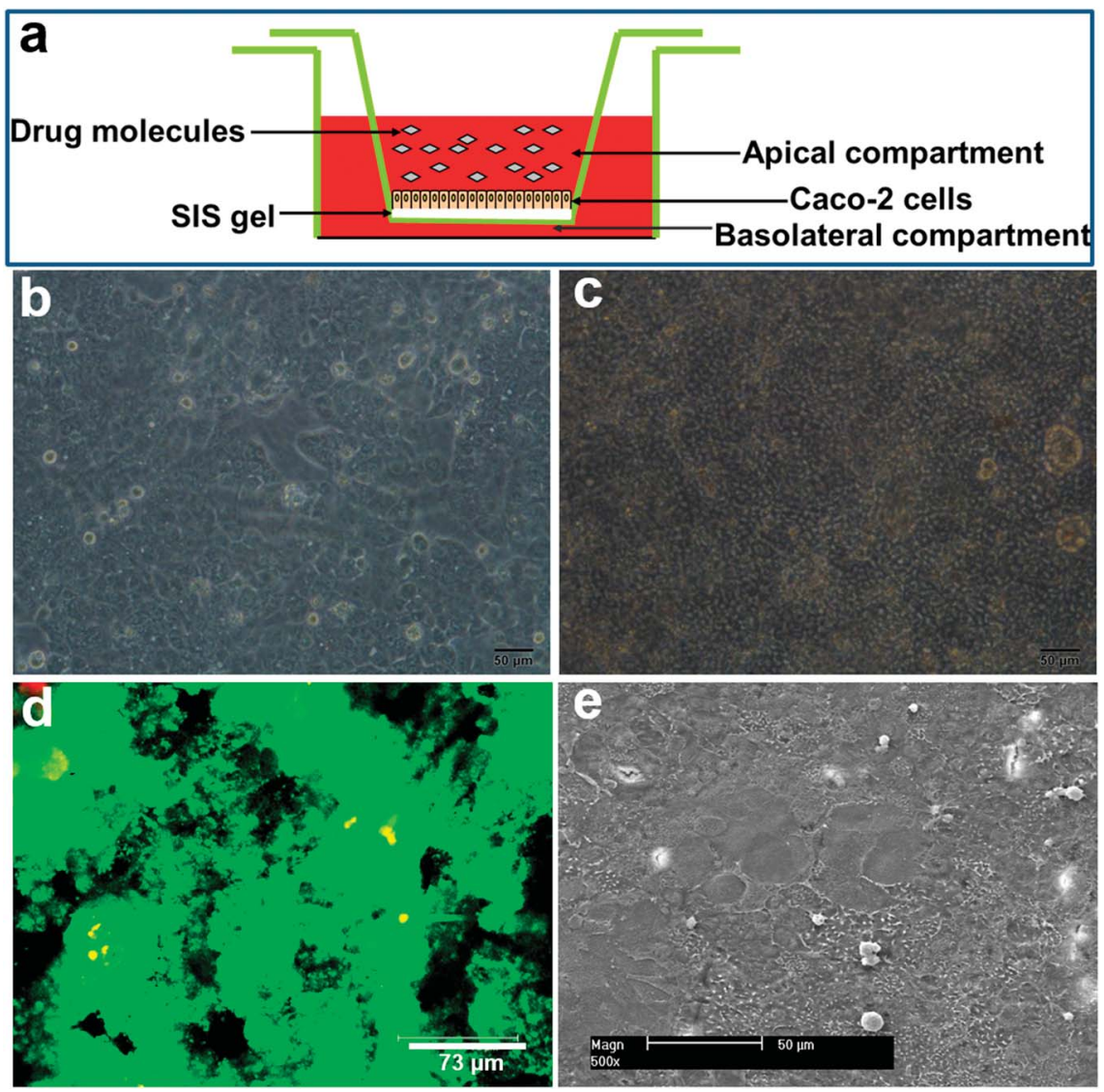

Fig. 2 Morphology and cell viability of the reconstructed fast model. Caco-2 cells seeded on the surface of the SIS hydrogel (a) were observed to be completely confluent on day 3 (b). A dense cell monolayer was generated on the top of the SIS hydrogel on day 7 (c). Live/dead staining showed that Caco-2 cells displayed good viability after one week of cultivation (d). The uniform topology of the epithelial layer formed on the top of SIS hydrogel was revealed by SEM imaging (e).

revealed by SEM imaging (Fig. 2e), supporting the intercellular tightness of Caco-2 cells grown on the surface of the SIS hydrogel.

\section{Proliferation ability and alkaline phosphatase activity of the newly developed model}

Consistent with what was observed in the morphological characterizations mentioned above, DNA quantification analysis provided further evidence for the SIS hydrogel in promoting Caco-2 cell proliferation. As shown in Fig. 3a, the DNA content of the cells in both the fast model and 21 day control model increased progressively during the first 11 days of cultivation and then started to gradually decrease, indicating that cell proliferation reached a plateau. Surprisingly, the proliferation rate of cells in the fast model was noticeably higher than that of the 21 day model throughout the culture period, and the most significant difference between them also appeared on day 11 .

To verify whether the SIS hydrogel could synchronously facilitate both Caco-2 cell proliferation and differentiation in the fast model, the activities of alkaline phosphatase (ALP) were measured by the hydrolysis assay of its specific substrate at different time points. As shown in Fig. $3 \mathrm{~b}$, there was no significant difference between the cell ALP activity in the fast model and that in the 21 day control model at any indicated time point, suggesting that the SIS hydrogel has the potential to facilitate Caco-2 cell differentiation towards matured intestinal epithelial cells during the 7 days of the cultivation period, thereby helping the fast model to achieve similar ALP activity to the traditional Caco-2 cell model.

\section{Cell differentiation and transport function of the newly developed model}

To further identify the potential role of SIS in accelerating the differentiation process of Caco-2 cells, the mRNA expression levels of intercellular tight proteins (ZO-1, occludin, E-cadherin, Claudin-1) and transporters (OATP1A2, PEPT1, P-gp, MRP2, BCRP) in both the fast model and the 21 day control model were examined by real-time RT-PCR. Subsequently, paracellular permeability and transporter-mediated active influx or efflux across the two models were determined to evaluate the functionality of the Caco-2 cell monolayers. As shown in Fig. 4a, there existed no significant difference between the ZO-1 and Claudin-1 mRNA expression levels in the fast model and those in the 21 day model. Strikingly, the transcript levels of occludin and E-cadherin in the fast model were even higher than in the 21 day-model. Furthermore, TEER values of the two models 

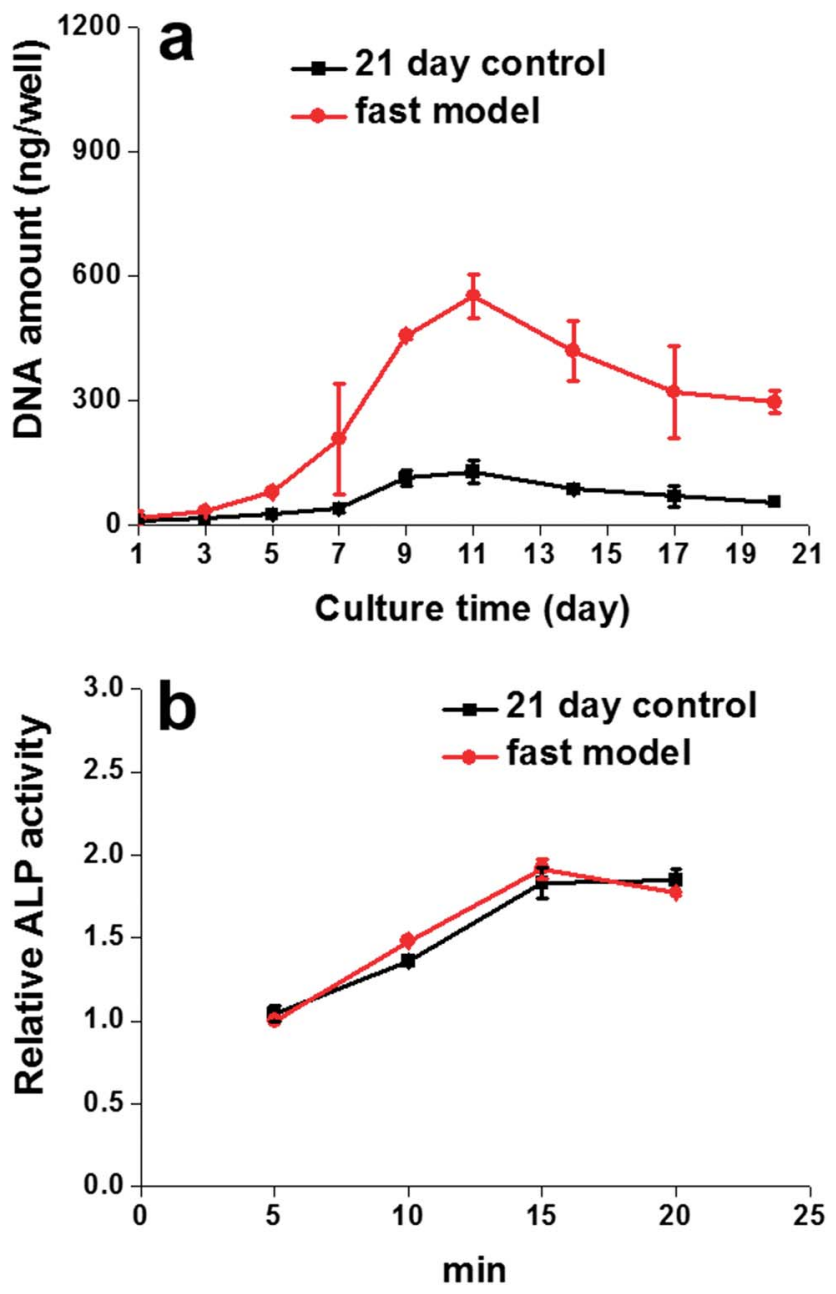

Fig. 3 Proliferation ability and ALP activity of the fast model. The proliferation rate of cells in the fast model was noticeably higher than that of the 21 day model throughout the culture period, and the most significant difference between them appeared on day 11 (a). There was no significant difference between the cell ALP activity in the fast model and that in the 21 day control model at any indicated time points (b).

were monitored throughout the cultivation period and the data showed that they both increased gradually until the appearance of the maximum values at $596 \pm 48 \Omega \mathrm{cm}^{2}$ and $1081 \pm 67 \Omega \mathrm{cm}^{2}$ in the fast model and 21 day control model, respectively (Fig. 4b). This result undoubtedly supports the desirable integrity of the epithelial layer generated by the reconstructed fast model, in spite of its relatively low level of TEER peak values compared to the traditional 21 day model. The integrity of the epithelium in the fast model was further evidenced by the paracellular permeability of fluorescein, a well-known marker compound for the paracellular pathway. The absorptive (apical to basolateral) $P_{\text {app }}$ value of fluorescein across the fast model was comparable with that across the 21 day model (Fig. 4c).

Transporters and their mediated active influx or efflux play another significant role in determining the intestinal permeability of oral drugs. As shown in Fig. 5a, no statistically significant difference in OATP1A2, PEPT1 and P-gp mRNA expression levels existed between the fast model and the traditional 21 day model. Consistent with the unchanged transcription levels, the three transporters (OATP1A2, PEPT1, P-gp) were observed to mediate no significant different absorptive permeability of their respective specific substrates (fexofenadine, cephalexin and digoxin) across the two models (Fig. 5b). More importantly, in comparison with the 21 day control model, the mRNA expression levels of two efflux transporters (MRP2 and BCRP) expressed at the apical side of the intestinal epithelial cells were significantly upregulated in the fast model (Fig. 5a). As expected, this result is consistent with the functionality evaluation of MRP2 and BCRP, in which lower absorptive $P_{\text {app }}$ values of their respective specific substrates (sulfasalazine and estradiol-17-G) were observed in the fast model compared with the 21 day model (Fig. 5b). These results strongly support the fact that the involvement of the SIS hydrogel in the Caco-2 cell model could facilitate functional cell differentiation and consequently accelerate the formation of Caco-2 cell monolayers.

\section{Gene expression and the activity of metabolizing enzymes in the fast model}

The gene expression level and catalytic activity of UGT1A1 responsible for the glucuronidation of drugs, a metabolism enzyme widely expressed in intestinal epithelial cells, were determined to further characterize the functional differentiation of the fast model. Intriguingly, the mRNA expression level of UGT1A1 in the fast model was 4.24 times higher than that in the 21 day control model (Fig. 6a). However, the overall UGT enzyme activity of the cells in the fast model did not significantly differ from that in the 21 day model as indicated by the unchanged metabolism of 4-methylumbelliferone (4-MU), a well-established UGT substrate (Fig. 6b). In any case, these results provide further evidence that the SIS hydrogel integrated into the fast model could contribute to functional Caco-2 cell differentiation.

The permeability of passively transported model compounds in the fast model

To further confirm the functionality of the reconstructed fast model, a series of passively transported marketed drugs $(n=19)$ with different absorption fractions in humans were selected and subjected to transport experiments. Their absorptive $P_{\text {app }}$ values were determined in the fast model as well as the traditional 21 day control model, and the two groups of $P_{\text {app }}$ values were both found to rank in the same order as their absorption fractions (Table 2). In order to clearly compare the transport function of the two models, the correlation analysis between the absorptive permeability of these model compounds in the fast model and that in the 21 day model was performed. As shown in Fig. 7, a desirable linear correlation between the two groups of absorptive $P_{\text {app }}$ values was observed, and the correlation coefficient $\left(R^{2}\right)$ was 0.9458 . This result further validated the fact that the developed fast model with the SIS hydrogel exhibited similar transport functionality to the conventional 21 day Caco2 cell model. 

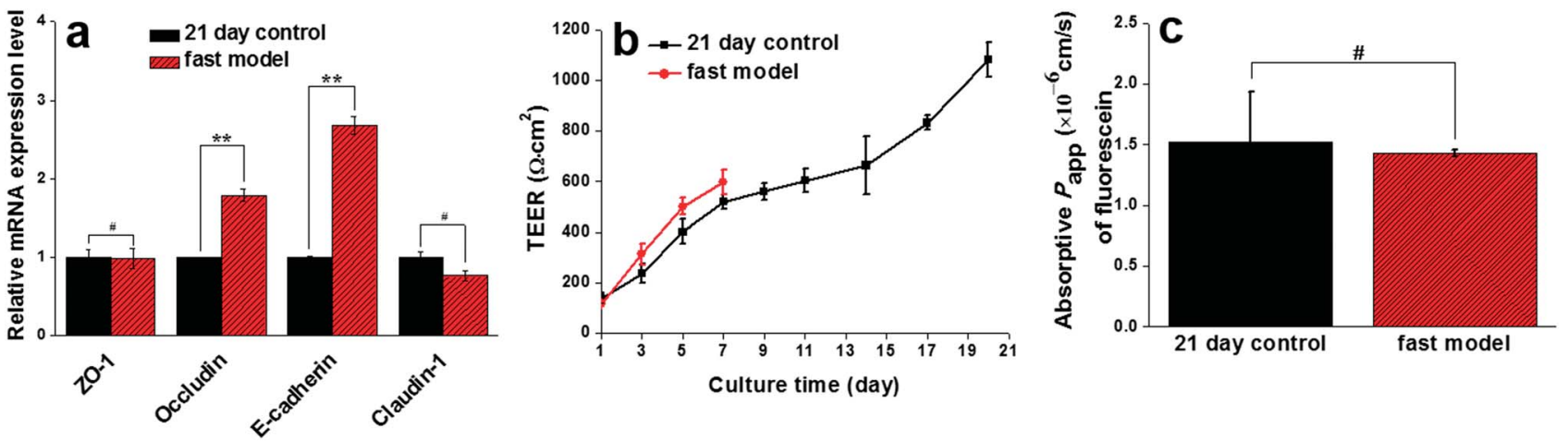

Fig. 4 Intercellular tight protein expression level, TEER values measurement, and paracellular permeability of the fast model. There was no significant difference between ZO-1 and claudin-1 mRNA expression levels in the fast model and those in the 21 day model (a), but the transcript levels of occludin and E-cadherin in the fast model were significantly higher than in the 21 day model. TEER values of both the fast model and the 21 day model increased gradually until the appearance of the maximum values at $596 \pm 48 \Omega \mathrm{cm}^{2}$ and $1081 \pm 67 \Omega \mathrm{cm}^{2}$, respectively (b), indicating the desirable integrity of the epithelial layer generated by the reconstructed fast model, in spite of its relatively low level of TEER peak values compared to the traditional 21 day model. The integrity of the epithelium in the fast model was further supported by the comparable paracellular permeability of fluorescein across the fast model to that across the 21 day model (c). Data are expressed as mean \pm SD $(n=3)$. ${ }^{\#} P>$ $0.05, * P<0.05, * * P<0.01, * * * P<0.001$

\section{Discussion}

The Caco-2 cell model has been generally regarded as a valuable permeability screening model that can be used in the lead optimization process of drug discovery, but at least 3 weeks of culture is a major obstacle to its access to high-throughput. To address this issue, the effective combination of native ECM from the small intestine (SIS) and the traditional Caco-2 cell model was performed to construct a novel fast screening model for drug absorption evaluation in the present study. To the best of our knowledge, the exact effect of SIS on Caco-2 cell proliferation and differentiation towards matured intestinal epithelial cells has never been well elucidated, despite the report that Schweinlin et al. have attempted to integrate the primary SIS sheet into the transwell to construct the intestinal tissue model. ${ }^{41}$ Our results have indicated that the SIS hydrogel not only exhibited the potential to promote Caco- 2 cell proliferation resulting in the fast generation of a fully confluent monolayer, but also significantly facilitated the cell differentiation and maturation in terms of similar AP activity, paracellular transport ability, absorptive transporters-mediated influx, and UGT-mediated metabolism to the 21 day model, as well as much higher excretive transportersmediated efflux than the conventional Caco-2 model. Furthermore, an excellent linear correlation was obtained between the $P_{\text {app }}$ values in the fast model and those in the conventional 21 day Caco- 2 model for drugs absorbed primarily by passive diffusion, which is responsible for the intestinal absorption of at least $80 \%$ of commercial drugs. ${ }^{42}$ In addition, it is highly worth mentioning that the native ECM incorporated into the fast model is from swine jejunum, thus allowing for its low cost and easy procurement. Hence, this study has not only advanced our knowledge of how SIS contributes to small intestinal epithelial monolayer formation but also provides the possibility for substituting the laborious and time-consuming Caco- 2 permeability assessment with the accelerated and cost-effective screening system based on decellularized ECM.
The fast cell growth profile presents a prerequisite for the formation of completely confluent and functional epithelial monolayer in the Caco-2 cell model. Distinct from the strategies applied in previous studies, ${ }^{\mathbf{1 4}}$ we have innovatively accomplished an enhanced cell proliferation through SIS hydrogel incorporation into the fast model evidenced by the higher total DNA content when compared with the traditional 21 day Caco- 2 cell model. This result is somewhat consistent with a previous report, where Ahn et al. reported that the SIS sheet could act as a potential scaffold to enhance the human bone marrow stem cells proliferation. ${ }^{43}$ Besides, it is elucidated that the proliferative activities of human umbilical vein endothelial cells or human bladder smooth muscle cells were also enhanced when the cells were exposed to SIS components. ${ }^{22}$ Direct interactions between bioactive ECM components such as collagen, fibronectin as well as growth factors preserved in SIS and cells might provide a reasonable explanation for the proliferative effect exerted by the decellularized ECM, but the detailed underlying mechanism still needs to be further explored. Overall, SIS is capable of offering a compatible environment to support the fast proliferation of intestinal epithelial cells, consequently enabling the development of an accelerated Caco- 2 permeability assay.

In contrast to the existing short-time Caco- 2 cell cultures that have to be compromised due to the incomplete formation of tight junctions and the inadequate expression of transporters despite their desirable proliferation ability, ${ }^{\mathbf{1 3}}$ the present accelerated screening model exhibits similar paracellular permeability and transporters-mediated influx or efflux to the conventional Caco- 2 cell model. Unexpectedly, the expression levels of excretive transporters (MRP2 and BCRP), as well as their respective transport abilities, were even significantly elevated as compared to the 21 day model. These results indicate that the developed 7 day fast model can not only be potentially utilized to evaluate drug permeability as a replacement for the 21 day model but can also be applied 

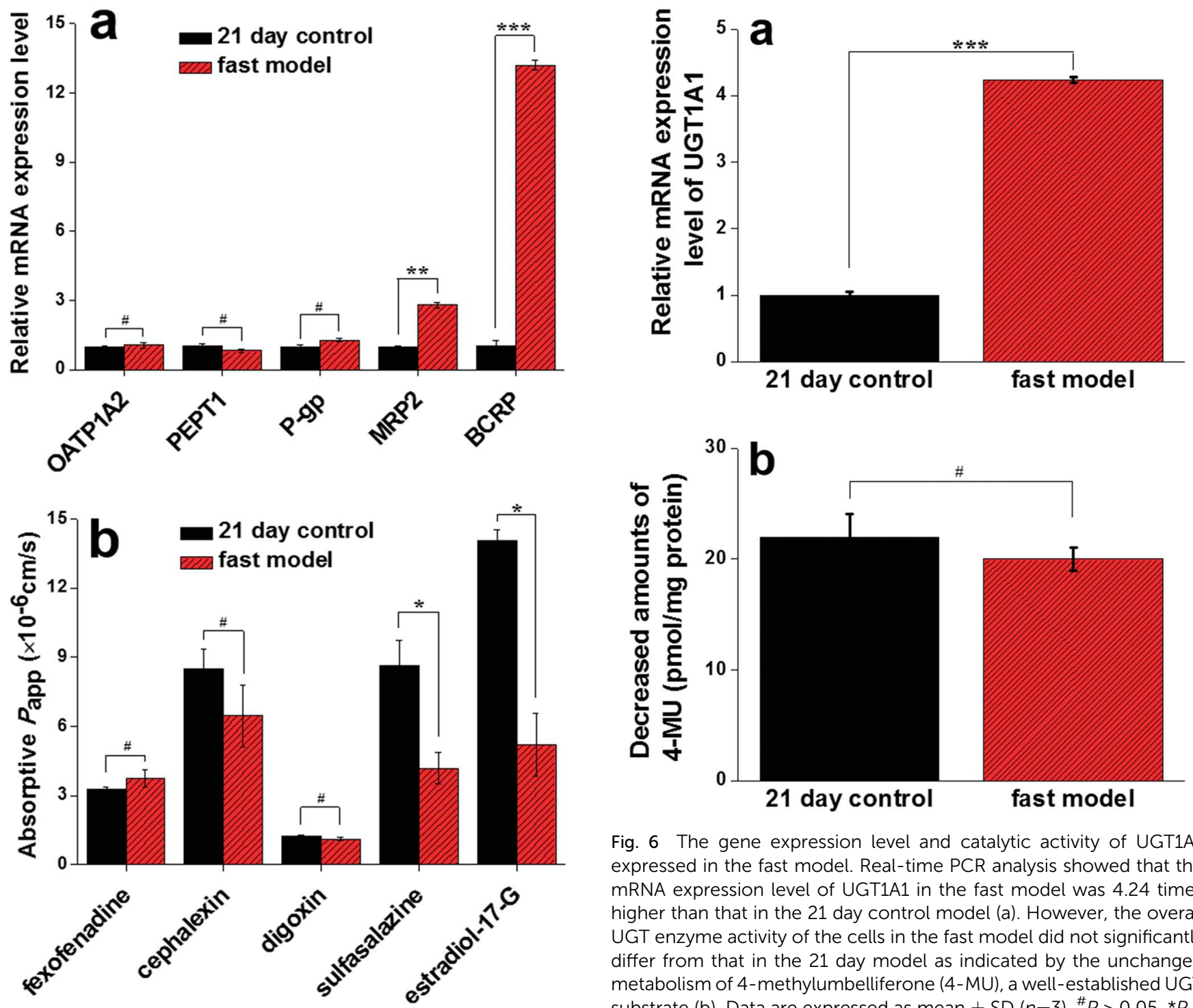

Fig. 6 The gene expression level and catalytic activity of UGT1A1 expressed in the fast model. Real-time PCR analysis showed that the mRNA expression level of UGT1A1 in the fast model was 4.24 times higher than that in the 21 day control model (a). However, the overall UGT enzyme activity of the cells in the fast model did not significantly differ from that in the 21 day model as indicated by the unchanged metabolism of 4-methylumbelliferone (4-MU), a well-established UGT substrate (b). Data are expressed as mean $\pm \mathrm{SD}(n=3)$. ${ }^{\#} P>0.05,{ }^{\star} P<$

Fig. 5 The mRNA expression level of transporters and their mediated substrate permeability in the fast model. No statistically significant difference in OATP1A2, PEPT1 and P-gp mRNA levels existed between the fast model and the traditional 21 day model (a). Consistent with the unchanged transcript levels, the three transporters (OATP1A2, PEPT1, and $\mathrm{P}$-gp) were observed to mediate no significantly different absorptive permeability of their respective specific substrates (fexofenadine, cephalexin, and digoxin) across the two models (b). Compared with the 21 day control model, the mRNA expression levels of two efflux transporters (MRP2 and BCRP) were significantly upregulated in the fast model (a). This result is consistent with the functionality evaluation of MRP2 and BCRP, in which the lower absorptive $P_{\text {app }}$ values of their respective specific substrates (sulfasalazine and estradiol-17-G) were observed in the fast model in comparison with the 21 day model (b). Data are expressed as mean $\pm \mathrm{SD}(n=3)$. ${ }^{\#} P>$ $0.05,{ }^{*} P<0.05,{ }^{*} P<0.01,{ }^{* *} P<0.001$.

efficaciously to screen substrates or inhibitors of MRP2 and BCRP. Regarding the improvement in paracellular transport, our findings are somewhat consistent with a report from Uchida $e t$ al. that the collagen coating on inserted systems made the cell monolayer tighter and accelerated the $0.05,{ }^{* *} P<0.01,{ }^{* * *} P<0.001$.

development of the Caco-2 cell model, ${ }^{14}$ suggesting the importance of collagen as the major component of ECM in intestinal epithelium formation. Furthermore, it has been documented that the epidermal growth factor (EGF)-mediated activation of the mitogen-activated protein kinase (MAPK) cascade results in a significant increase in BCRP expression in multiple cell types at both the mRNA and protein levels, ${ }^{44,45}$ which is consistent with our results indicating the potent inductive effect on BCRP exerted by EGF as a major growth factor in decellularized SIS. ${ }^{43}$ Likewise, insulin-like growth factor-I (IGF-I), another growth factor preserved in decellularized SIS, ${ }^{23,43}$ was also demonstrated to upregulate MRP2 and BCRP in the ovarian cancer cell line. ${ }^{46}$ In addition, Kondo et al. revealed that the induced mRNA level of UGT1A1 in response to omeprazole was much higher in human induced pluripotent stem (iPS) cells cultured on Matrigel as compared to the cells on collagen I, which may be attributed to laminin, a main component of Matrigel. ${ }^{47}$ Thus, the potent induced effect of 


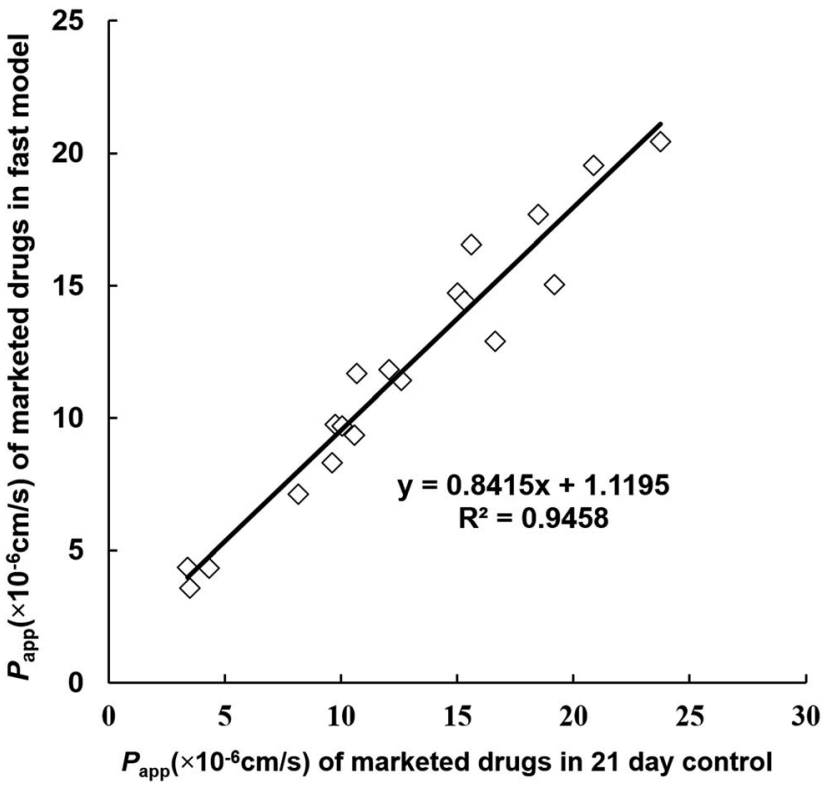

Fig. 7 The correlation analysis between the absorptive permeability of 19 model compounds in the fast model and that in the 21 day model. A desirable linear correlation between the two groups of absorptive $P_{\text {app }}$ values was observed, and the correlation coefficient $\left(R^{2}\right)$ was 0.9458 . This result further supported the fact that the reconstructed fast model with SIS hydrogel exhibited similar transport functionality to the conventional 21 day Caco- 2 cell model.

the SIS hydrogel on UGT1A1 expression level demonstrated in the current study might also be due to the rich laminin content in native SIS. Besides, it was observed that hepatocytes on soft substrates showed higher gene expression of UGT1A1; ${ }^{48}$ thus, substrate stiffness softening from transwell insert to SIS gel might provide another reasonable explanation for the augmented UGT1A1 expression level in the fast model in comparison with the traditional Caco-2 cell model. The unchanged metabolism activity of UGT observed in the fast model compared to the conventional Caco- 2 model can be explained by the fact that the UGT enzyme family encompasses lots of members and the increased expression of the single gene (UGT1A1) might not be sufficient to modulate the overall UGT activity. ${ }^{39}$ Taken together, these findings do not only implicate the importance of various constituents in native ECM in facilitating the differentiation of intestinal epithelial cells, but they also underscore the feasibility of employing the 7 day fast model to assess drug intestinal permeability.

\section{Conclusions}

The accelerated 7 day screening model based on native decellularized ECM was shown to provide a valuable and costeffective alternative to the traditional 21 day Caco- 2 model for accurate drug absorption prediction. Thus, it is hoped that this novel physiological assay system would potentially work as a reliable high-throughput tool for lead optimization in future drug discovery.

\section{Conflicts of interest}

There are no conflicts of interest to declare.

\section{Acknowledgements}

This work was supported by the National Key Research and Development Program of China (2017YFC1700200, 2017YFC1702000), the NSF of China (81473181, 81773687, 81703604, 81672961 and 81573501), Program of Shanghai Academic/Technology Research Leader (18XD1403600), the National Scientific and Technological Major Projects of China (2017ZX09101004), the Innovative Entrepreneurship Program of High-level Talents in Dalian (2016RQ025 \& 2017RQ121).

\section{References}

1 R. L. DiMarco, D. R. Hunt, R. E. Dewi and S. C. Heilshorn, Biomaterials, 2017, 129, 152-162.

2 P. A. Bilat, E. Roger, S. Faure and F. Lagarce, Drug Discovery Today, 2017, 22, 761-775.

3 N. Ward, J. Gastrointest. Surg., 2010, 14, 1045-1051.

4 J. J. Yu, R. L. Carrier, J. C. March and L. G. Griffith, Drug Discovery Today, 2014, 19, 1587-1594.

5 N. Li, D. D. Wang, Z. G. Sui, X. Y. Qi, L. Y. Ji, X. L. Wang and L. Yang, Tissue Eng., Part C, 2013, 19, 708-719.

6 R. Joubert, J. D. Steyn, H. J. Heystek, J. H. Steenekamp, J. L. Du Preez and J. H. Hamman, Expert Opin. Drug Delivery, 2017, 14, 179-187.

7 F. G. Pan, L. Han, Y. Zhang, Y. D. Yu and J. B. Liu, Int. J. Food Sci. Nutr., 2015, 66, 680-685.

8 B. Yi, K. Y. Shim, S. K. Ha, J. Han, H. H. Hoang, I. Choi, S. Park and J. H. Sung, BioChip J., 2017, 11, 219-232.

9 S. H. Kim, M. Chi, B. Yi, S. H. Kim, S. Oh, Y. Kim, S. Park and J. H. Sung, Integr. Biol., 2014, 6, 1122-1131.

10 H. Li, J. Li, L. Liu, Y. C. Zhang, Y. L. Luo, X. L. Zhang, P. Yang, M. N. Zhang, W. F. Yu and S. Qu, Planta Med., 2016, 82, 12021207.

11 A. Beloqui, A. des Rieux and V. Preat, Adv. Drug Delivery Rev., 2016, 106, 242-255.

12 S. Yamashita, K. Konishi, Y. Yamazaki, Y. Taki, T. Sakane, H. Sezaki and Y. Furuyama, J. Pharm. Sci., 2002, 91, 669-679.

13 E. Sevin, L. Dehouck, A. Fabulas-da Costa, R. Cecchelli, M. P. Dehouck, S. Lundquist and M. Culot, J. Pharmacol. Toxicol. Methods, 2013, 68, 334-339.

14 M. Uchida, T. Fukazawa, Y. Yamazaki, H. Hashimoto and Y. Miyamoto, J. Pharmacol. Toxicol. Methods, 2009, 59, 39-43.

15 J. Alsenz and E. Haenel, Pharm. Res., 2003, 20, 1961-1969.

16 G. R. Fercana, S. Yerneni, M. Billaud, J. C. Hill, P. VanRyzin, T. D. Richards, B. M. Sicari, S. A. Johnson, S. F. Badylak, P. G. Campbell, T. G. Gleason and J. A. Phillippi, Biomaterials, 2017, 123, 142-154.

17 R. H. Dosh, N. Jordan-Mahy, C. Sammon and C. L. Le Maitre, Tissue Eng., Part B, 2018, 24, 98-111.

18 C. M. Howard and T. A. Baudino, J. Mol. Cell. Cardiol., 2014, 70, 19-26. 
19 S. L. K. Bowers and T. A. Baudino, Birth Defects Res., Part C, 2010, 90, 1-7.

20 V. Visco, F. A. Bava, F. D'Alessandro, M. Cavallini, V. Ziparo and M. R. Torrisi, J. Cell. Physiol., 2009, 220, 204-213.

21 M. Parmaksiz, A. E. Elcin and Y. M. Elcin, J. Tissue Eng. Regen. Med., 2017, 11, 1754-1765.

22 B. Yang, L. H. Zhou, Z. Y. Sun, R. Yang, Y. Chen and Y. T. Dai, J. Biomed. Mater. Res., Part A, 2010, 93, 1100-1109.

23 M. Li, C. Zhang, M. J. Cheng, Q. Q. Gu and J. Y. Zhao, Mater. Sci. Eng., C, 2017, 75, 149-156.

24 G. S. Hussey, T. J. Keane and S. F. Badylak, Nat. Rev. Gastroenterol. Hepatol., 2017, 14, 540-552.

25 C. W. Chang, T. Petrie, A. Clark, X. Lin, C. S. Sondergaard and L. G. Griffiths, PLoS One, 2016, 11, e0153412f.

26 K. Lindberg and S. F. Badylak, Burns, 2001, 27, 254-266.

27 T. J. Keane, R. Londono, N. J. Turner and S. F. Badylak, Biomaterials, 2012, 33, 1771-1781.

28 S. Ferruzza, C. Rossi, M. L. Scarino and Y. Sambuy, Toxicol. in Vitro, 2012, 26, 1247-1251.

29 H. Bergmann, D. Rogoll, W. Scheppach, R. Melcher and E. Richling, Mol. Nutr. Food Res., 2009, 53, 1211-1225.

30 V. U. Buck, R. Windoffer, R. E. Leube and I. Classen-Linke, Histochem. Cell Biol., 2012, 137, 777-790.

31 J. Moldvay, M. Jackel, C. Paska, I. Soltesz, Z. Schaff and A. Kiss, Lung Cancer, 2007, 57, 159-167.

32 S. Seki, M. Kobayashi, S. Itagaki, T. Hirano and K. Iseki, Biochim. Biophys. Acta, 2009, 1788, 911-917.

33 N. Maubon, M. Le Vee, L. Fossati, M. Audry, E. Le Ferrec, S. Bolze and O. Fardel, Fundam. Clin. Pharmacol., 2007, 21, 659-663.

34 X. D. Wang, M. X. Meng, L. B. Gao, T. Liu, Q. Xu and S. Zeng, Int. J. Pharm., 2009, 378, 1-8.

35 W. Brand, P. A. I. van der Wel, M. J. Rein, D. Barron, G. Williamson, P. J. van Bladeren and I. Rietjens, Drug Metab. Dispos., 2008, 36, 1794-1802.
36 J. R. Bacon, G. Williamson, R. C. Garner, G. Lappin, S. Langouet and Y. P. Bao, Carcinogenesis, 2003, 24, 19031911.

37 V. Uskokovic, P. P. Lee, L. A. Walsh, K. E. Fischer and T. A. Desai, Biomaterials, 2012, 33, 1663-1672.

38 N. Li, D. D. Wang, G. B. Ge, X. L. Wang, Y. Liu and L. Yang, Planta Med., 2014, 80, 290-296.

39 H. Bothe, K. Gassmann, C. Gotz, E. Fritsche, J. Abel and T. Haarmann-Stemmann, Toxicol. Lett., 2011, 203, 258-264.

40 S. Skolnik, X. N. Lin, J. L. Wang, X. H. Chen, T. He and B. L. Zhang, J. Pharm. Sci., 2010, 99, 3246-3265.

41 M. Schweinlin, A. Rossi, N. Lodes, C. Lotz, S. Hackenberg, M. Steinke, H. Walles and F. Groeber, Drug Delivery Transl. Res., 2017, 7, 217-227.

42 E. H. Kerns, L. Di, S. Petusky, M. Farris, R. Ley and P. Jupp, J. Pharm. Sci., 2004, 93, 1440-1453.

43 H. H. Ahn, K. S. Kim, J. H. Lee, M. S. Lee, I. B. Song, M. H. Cho, Y. N. Shin, M. S. Kim, G. Khang and H. B. Lee, Int. J. Biol. Macromol., 2007, 41, 590-596.

44 Y. Imai, K. Ohmori, S. Yasuda, M. Wada, T. Suzuki, K. Fukuda and Y. Ueda, Cancer Sci., 2009, 100, 1118-1127.

45 M. Jani, C. Ambrus, R. Magnan, K. T. Jakab, E. Beery, J. K. Zolnerciks and P. Krajcsi, Arch. Toxicol., 2014, 88, 1205-1248.

46 N. Benabbou, P. Mirshahi, M. Cadillon, J. Soria, A. Therwath and M. Mirshahi, Int. J. Oncol., 2013, 43, 685-694.

47 Y. Kondo, T. Iwao, K. Nakamura, T. Sasaki, S. Takahashi, N. Kamada, T. Matsubara, F. J. Gonzalez, H. Akutsu, Y. Miyagawa, H. Okita, N. Kiyokawa, M. Toyoda, A. Umezawa, K. Nagata, T. Matsunaga and S. Ohmori, Drug Metab. Pharmacokinet., 2014, 29, 237-243.

48 V. Natarajan, E. J. Berglund, D. X. Chen and S. Kidambi, RSC Adv., 2015, 5, 80956-80966. 